\title{
Coupled Inductor Based Radio Energy Transmission Circuit
}

\author{
Xuan Wu \\ School of North China Electric Power University, Baoding 071000, China. \\ 3336180262@qq.com
}

\begin{abstract}
The wireless energy transmission based on coupled inductor is a new type of power transmission technology, which can overcome many shortcomings of traditional power transmission methods. This technology can provide strong support for the further development of modern society and bring great convenience to people's lives. Therefore, research on the coupling energy-based radio energy transmission circuit will have important academic significance and practical value. This paper first introduces the development history of radio energy transmission technology and the current development. Then, the paper analyzes the circuit structure of the wireless energy transmission system based on inductive coupling in detail, studies its working principle, uses the mutual inductance model to model the circuit of the wireless energy transmission system and calculate the relevant transmission efficiency. In the circuit modeling process, the selection and calculation of the compensation capacitor is studied emphatically, and further parameters are calculated for the circuit after the compensation is improved. After that, this paper studies the characteristics of the magnetically coupled resonant radio energy transmission method in detail, and focuses on the difference and connection between the magnetic coupling resonant type and the inductive coupling type.
\end{abstract}

Keywords: Coupling inductance, capacitance compensation, magnetic coupling resonance, relay coil, mutual inductance, offset angle.

\section{Introduction}

Wireless Power Transmission (WPT), also known as Inductively Coupled Power Transfer (ICPT), uses the transmitter and receiver to transfer energy at a certain distance. The transmission of electrical energy without electrical connection. At present, wireless energy transmission technology has become a hot issue at home and abroad. And has been applied in the field of wireless charging.

\section{Circuit Model of Inductively Coupled Wireless Energy Transmission System}

\subsection{System Composition.}

The wireless energy transmission system based on the principle of inductive coupling is mainly composed of four parts, namely an $\mathrm{AC}$ power source, a primary side converter, a separable transformer and a secondary side converter. Its system structure diagram is shown in Figure 2-1. Compared with the traditional power transmission system, the system adds a primary side converter and a secondary side converter, the purpose of which is to change the operating frequency of the system. Since the radio energy transmission system is a loose coupling system, its coupling coefficient is not high, so the transmission performance is generally poor. In order to improve the transmission capacity of the system, the primary side converter is used to convert the AC power of the power frequency into a high frequency power supply. The separable transformer is the most important component of the contactless power transmission system, and its performance directly affects whether the entire system can work stably and efficiently. The advantage of the wireless energy transmission system based on the inductive coupling principle is that the system is simple and efficient at close range, but its transmission efficiency decreases sharply with increasing distance 


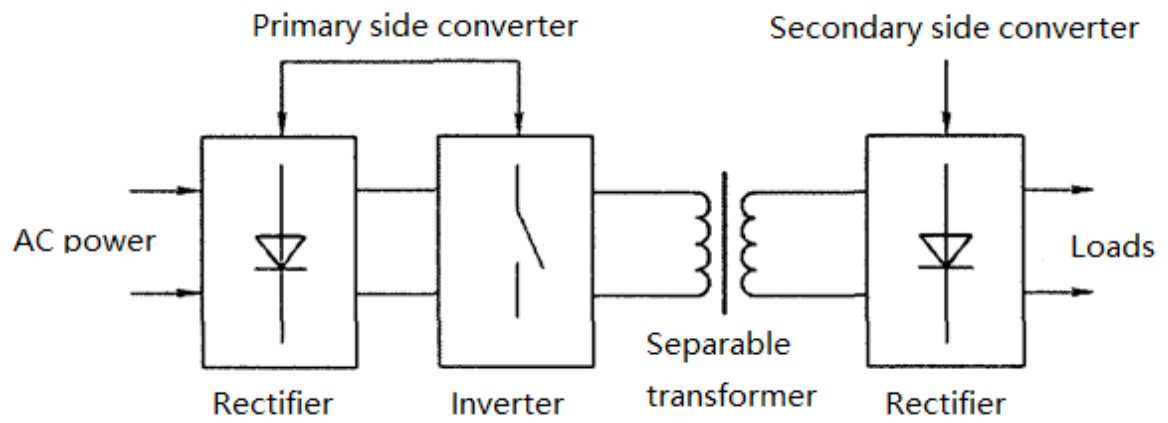

Fig. 1 Inductively coupled wireless energy transmission system structure

In the inductively coupled wireless energy transmission system, the mutual inductance coil or the loosely coupled transformer is the core part, and its circuit model is essentially a mutual inductance model. The electrical energy is coupled through the mutual inductance system to achieve wireless transmission between the two sides. Quantitative analysis of inductively coupled radio energy transmission systems is possible based on circuit theory. Because based on circuit theory modeling, parameters such as voltage, current, and power can be easily calculated, which is in line with our needs for analyzing circuit performance.

Inductively coupled power wireless transmission system is mainly composed of four parts: power supply, transmitter, receiver and load, as shown in Figure 2-2. Its essence is a mutual inductance system. The biggest difference from the traditional transformer is that there is a relatively large air gap between the primary and secondary coils of the system.

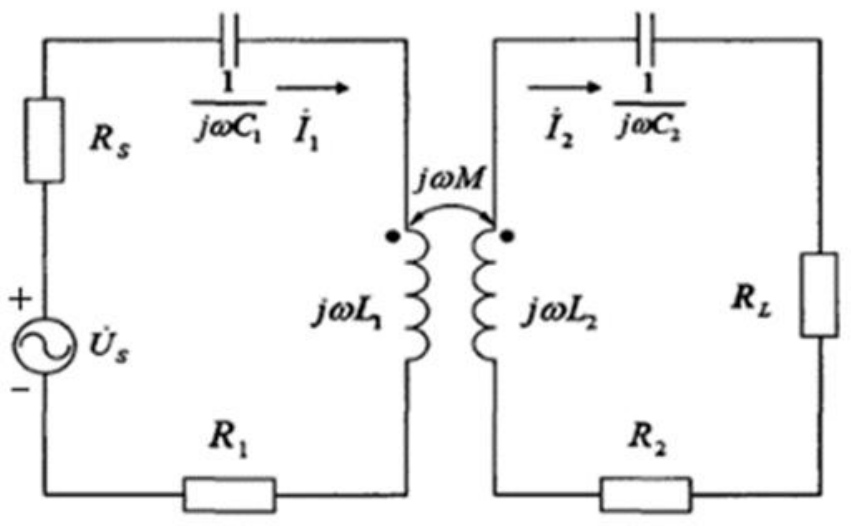

Fig. 2 Inductively coupled wireless energy transmission system schematic

In this system, the power source sends a signal generated to the transmitting coil L1. The transmitting coil then transmits energy to the receiving coil L2 by electromagnetic induction coupling, and the energy is transmitted from the transmitter to the receiver at a certain distance, and further transmitted to the load to complete the wireless transmission task of the energy.

In the mutual inductance model, the coils are used as a pair of inductors coupled to each other, and the input and output variables are solved by establishing a constraint equation for the primary and secondary resistance inductances. If the compensation capacitance is taken into account, it is necessary to add a capacitance component to the circuit equations on the primary side and the secondary side.

\subsection{Mutual Inductance Circuit Model.}

Inductively coupled radio energy transmission systems have poor coupling performance due to air gaps. The coupling coefficient is usually below 0.8 , and some even less than 0.1 , and the leakage inductance is large. In view of this, we decided to use the mutual inductance model to study the 
inductively coupled wireless energy transmission system. In the mutual inductance model, it is not necessary to consider the mutual inductance and the leakage inductance separately, and the concept of reflecting the impedance is used to describe the coupling between the primary and secondary coils, and then the total influence of the secondary side on the primary side can be expressed. This is also the advantage of the mutual inductance model.

The coupling coefficient of the primary coil is defined as:

$$
\mathbf{k}=\frac{M}{\sqrt{L_{1} L_{2}}}
$$

A primary coil and disposed respectively $\mathrm{R}_{1}$ resistance and $\mathrm{L}_{1}$, the secondary coil inductance and resistance are disposed as $R_{2}$ and $L_{2}$. Mutual inductance is $M$. Let the load connected to the secondary side be a pure resistive load $\mathrm{R}_{\mathrm{L}}$. The current on the primary side is $\dot{I}_{1}$, the current on the secondary side is $\dot{I}_{2}$, and the power supply is $U_{S}$. Then $j \omega M \dot{I}_{1}$ is the induced voltage value of the primary current on the secondary side, and $\mathrm{j} \omega \mathrm{MI}_{2}$ is the induced voltage value of the secondary current on the primary side. According to the positive direction given in the figure, the influence of the compensation capacitor is temporarily ignored by the KVL equation. The circuit equations of the primary and secondary loops are:

$$
\begin{gathered}
\dot{U}_{S}=\mathrm{j} \omega L_{1} \dot{I}_{1}+R_{1} \dot{I}_{1}-j \omega M \dot{I}_{2} \\
\mathrm{j} \omega M \dot{I}_{1}=j \omega L_{2} \dot{I}_{2}+\left(R_{2}+R_{L}\right) \dot{I}_{2}
\end{gathered}
$$

According to equations (2) and (3), the equivalent model of the circuit can be obtained, as shown in Fig. 3 and Fig. 4.

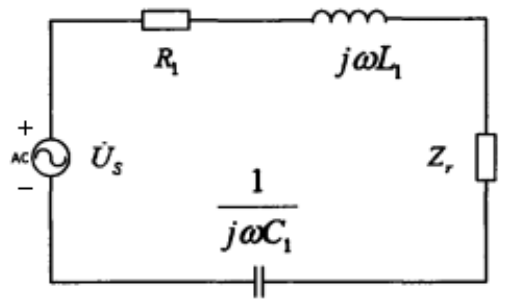

Fig. 3 Equivalent circuit seen by the primary port

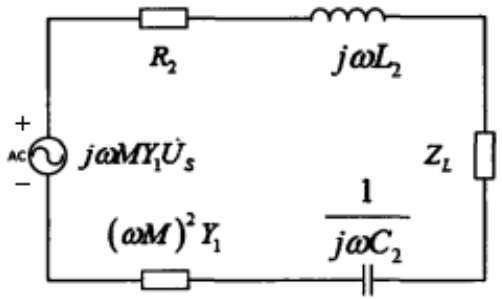

Fig. 4 Equivalent circuit seen by the secondary port

\section{Chapter III Working Principle of Magnetically Coupled Resonance System}

The basic principle of magnetically coupled resonant wireless power transmission is that at a certain coupling distance, by having two resonant coils of the same frequency, resonance occurs at high frequencies through magnetic field coupling, resulting in a time-varying high-intensity change centered on the coil. The magnetic field and the coupling coil continuously perform energy conversion to realize wireless energy transmission. The key point is that high-efficiency energy transmission can be realized when the system is in an object resonant at the same frequency. The specific schematic diagram is shown in the figure. In the research process of this paper, the parasitic capacitance of the coil itself is difficult to meet the resonant frequency required by the system. 


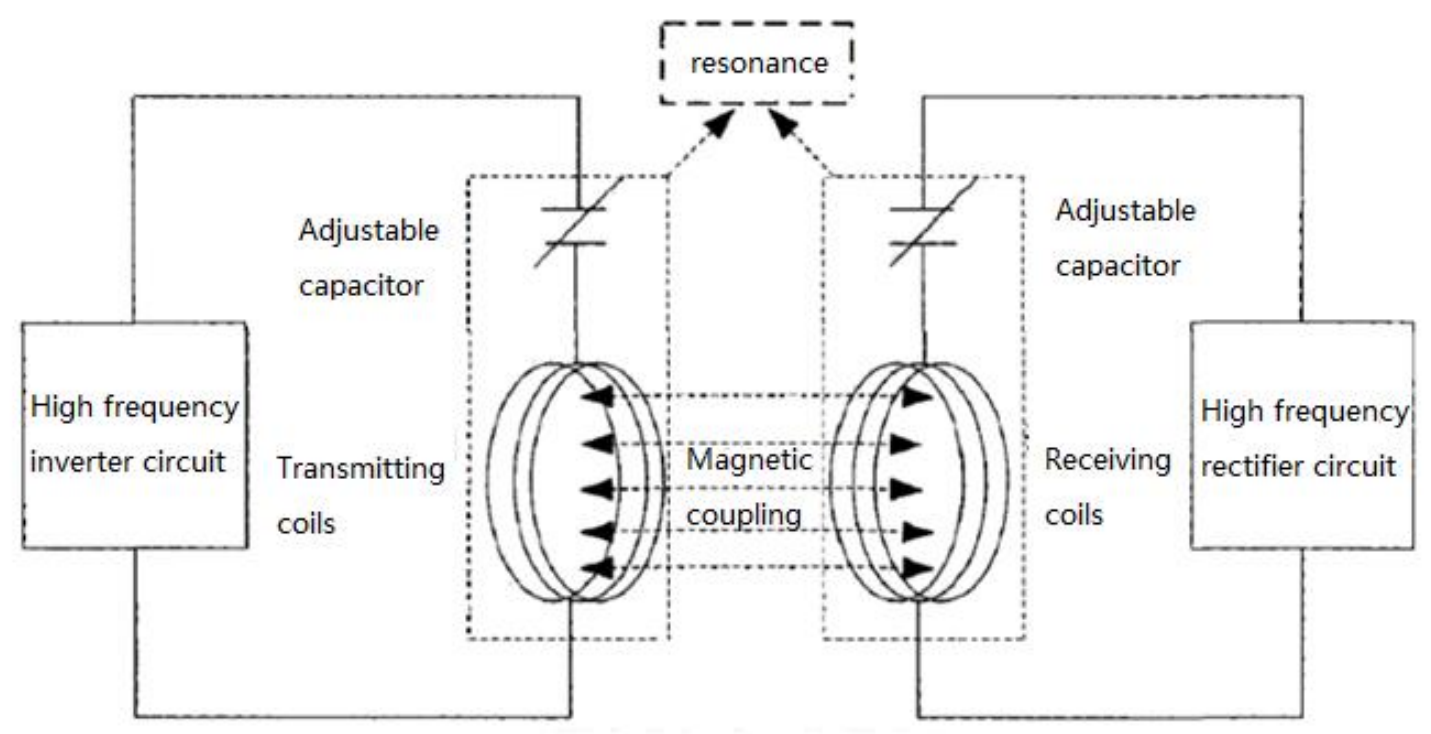

Fig. 5 Magnetic coupling resonant wireless power supply schematic

Therefore, by adjusting the external capacitor to make the system reach the required resonant frequency, the current is generated by the high-frequency inverter circuit. The magnetic field flows through the coil to generate a magnetic field. If a larger magnetic field is excited when the transmitting end resonates, the receiving end will generate an electric field through the magnetic field change through magnetic coupling. By adjusting the external resonant capacitor at the receiving end, the receiving end loop generates the same frequency as the transmitting end. Resonance, thus achieving maximum energy transfer of the magnetically coupled resonant wireless power supply. In order to make the system transmit energy efficiently, the system must be consistent in frequency, and must meet the basic conditions of magnetically coupled resonant wireless power supply.

\section{References}

[1]. A P Sample, D A Meyer, J R Smith. Experimental Results and Range Adaptation of Magnetically Coupled Resonators for Wireless Power Transfer [J], IEEE Transactions on Industrial Electronics, 2011, 58(2):544-554.

[2]. Sanghoon C, Yong-Hae K, Seung-Youl K, et al. Circuit-Model-Based Analysis of a Wireless Energy-Transfer System via Coupled Magnetic Resonances [J]. IEEE Transactions on Industrial Electronics. 2011, 58(7):2906-2914.

[3]. Liu Fuxin, Yang Yong, Jiang Dan, et al. Modelling and optimization of magnetically coupled resonant wireless power transfer system with varying spatial scales [J]. IEEE Transactions on Power Electronics, 2016,32(4):3240 - 3250. 